\title{
Group Work Intervention F Study on Adaptation of Left-behind Children's Schools in Senior Primary School
}

\author{
Qiu Yanling ${ }^{1, \text { a }}$, Zhang Gen ${ }^{2}$ \\ ${ }^{1}$ Department of Social Work, Yunnan Agricultural University, Panlong, Kunming, Yunnan, China \\ ${ }^{2}$ Department of Social Work, Yunnan Agricultural University, Panlong, Kunming, Yunnan, China \\ adesigner58@163.com
}

\begin{abstract}
In this article, by issuing a questionnaire adapted from the School Adaptation Scale for Primary Schools, we understand the adaptation status of left-behind children in grades 4 to 6 in terms of learning adaptation, interpersonal relationship adaptation, and psychological and emotional adaptation. Through pre-investigation analysis, a formal questionnaire is formed. Using SPSS analysis to analyze the adaptation status of left-behind children, it is found that there are maladaptation problems $\mathrm{F}$ left-behind children in grade 4 to 6 , in which interpersonal relationship and psychological and emotional aspects are especially serious. Therefore, through six growth group activities from the interpersonal and psychological emotional two aspects of intervention, focus on improving self-confidence, improve interpersonal skills, reduce loneliness, and then help left-behind children better adapt to school life. The data before and after the activity found that the self-confidence of left-behind children was significantly improved, interpersonal communication changed from passive to active, and school adaptation was significantly improved.
\end{abstract}

Keywords : group work, left-behind children, school adaptation.

\section{RESEARCH BACKGROUND}

According to the data of the sixth census in China in 2010 , the number of left-behind children in rural areas in China is about 61.02 million, accounting for $37.7 \%$ of the rural children. The social problems exposed by leftbehind children have been paid attention to by scholars. However, at present, domestic scholars pay more attention to education and personal safety of left-behind children, but ignore the problems of school adaptation of left-behind children. School is the second place for children, so whether school adaptation directly affects children's social integration. This paper studies the school adaptation of left-behind children at three levels: learning, interpersonal relationship and psychology and emotion. By issuing the adapted school adaptation scale, the data were collected to analyze and understand the problems of left-behind children in the fourth to sixth grades of F primary school, and to find out their needs. Based on the theory of advantage perspective, we mainly guide left-behind children to discover their own advantages, correctly understand themselves, further improve interpersonal relationships and emotional problems, improve the problems of poor school adaptation, and promote the healthy growth of leftbehind children.

\section{DOMESTIC AND FOREIGN STUDIES}

\subsection{Conceptual Study on Left-behind Children and School Adaptation}

School adaptation is a concept with broad extension and multiple dimensions. Most domestic scholars adopt the definition of Ladd. He believes that school adaptation is to participate in school activities happily in school and achieve academic success. Zou Hong divides school adaptation into learning adaptation and non-learning adaptation. Learning adaptation refers to maintaining good learning motivation and attitude under normal teaching life. At present, many scholars in China have different concepts of left-behind children, among which Chen Keyan defines left-behind children from four dimensions: definition, age, scale and region. However, in general, most scholars believe that left-behind children refer to minors under 18 years of age, two parents or one of them working outside the home, and are taken care of 
by one of the parents or other guardians, and cannot live with their parents for 6 months or more.

\subsection{Study on Adaptation of Left-behind Children's Schools in China}

Zhou Zongkui and others found that the school adaptation of left-behind children is not good, mainly manifested in learning adaptation; in addition, Yang Qin's research shows that left-behind children's academic adaptation is mainly reflected in late, absenteeism and other bad behavior, as well as pre-class, unable to do homework without asking teachers and students. Xiang Yinhui pointed out that because parents are away for a long time, the inner emotions of left-behind children have no place to vent, so the mood fluctuates greatly, which is more likely to cause inferiority complex, closure and other personality. Foreign scholar Matzukato found that left-behind children are more difficult to adapt to psychological emotion and behavior than non-leftbehind children. Li Jinghui's study of left-behind children in rural primary schools found that their learning adaptation scores and mental health were low, and their interpersonal communication was not optimistic. Fan Xianzuo believes that the poor interpersonal adaptation of left-behind children is due to the lack of family, parents' care leading to inferiority complex, closed and other psychological problems, thus affecting the communication between left-behind children. In addition, Zhou Yulin and Yang Yulan found that the poor interpersonal adaptation of left-behind children was passive in the process of communication, afraid to actively communicate and play with their peers, rarely participated in collective activities, and had little contact with teachers. At present, there is little research on leftbehind children in foreign countries, and there is no attention to the problem of school adaptation, so there are few references. The research level of domestic scholars is mainly focused on learning adaptation and interpersonal adaptation, and the other levels are less involved. This paper not only studies the school adaptation of left-behind children from the aspects of learning adaptation and interpersonal adaptation. Psychological and emotional adaptation was also studied, and group intervention was carried out.

\section{SCALE ESTABLISHMENT AND ANALYSIS}

\subsection{Scale Preparation and Revision}

In order to study the school adaptation of left-behind children in primary schools, a questionnaire was designed based on the middle school students' school adaptation scale and self-made questions. The reliability and validity of the relevant questions were not tested, so it was necessary to carry out pre-investigation analysis. And then form the final formal questionnaire. This paper randomly selected 30 left-behind children from grade four to grade six by simple random sampling, distributed primary school adaptation questionnaire, and carried out project analysis. The total correlation of the questions was eliminated A3、A5、B1、B5、B6、B7、C1、C2、 $\mathrm{C} 6$, and the reliability test value was 0.827 and 0.957 respectively. Form a formal questionnaire.

\subsubsection{Reliability and Validity Test}

A class in grades 4 to 6 was randomly selected for formal questionnaire analysis for all left-behind children in three classes. The reliability of the formal questionnaire was between $0.8-1$, indicating that the reliability was good, and the structural validity of the questionnaire was very good. The KMO value was 0.845 and the cumulative variance contribution rate was 77.029.

\subsubsection{Adaptation of Left-behind Children's Schools}

Frequency statistics of demographic variables in the questionnaire, $\mathrm{F}$ understand the basic situation of leftbehind children in the three classes of grade 4 to 6 of primary school, Table 1 shows 56 persons in the fourth grade, There are 43 in fifth grade, There are 51 persons in the sixth grade; There are 79 boys, 71 girls, Gender distribution is uniform; With 64 parents working in the field, One of the parents worked outside 86; There are 41 people who think they're extroverted, There are 109 introverts, $72.7 \%$, It shows that most left-behind children think they are introverted. The scale uses five grades, Always, often, sometimes, rarely, and never, Score $1,2,3,4$ and 5 respectively. The higher the score, The better the adaptation, Conversely, The worse the adaptation. Table 2 shows that the learning adaptation dimensions are divided into 2.815 , The dimensions of interpersonal adaptation were 2.767 , The dimensions of psychological and emotional adaptation were 2.561, Between 2-3, It shows that learning adaptation, interpersonal relationship adaptation, psychological and emotional adaptation are poor.

Table 1. Score of items in the scale

\begin{tabular}{cc}
\hline Code & Mean \\
\hline learning adaptation & 2.815 \\
interpersonal adaptation & 2.767 \\
psychological and & 2.561 \\
emotional adaptation & \\
\hline
\end{tabular}

\subsubsection{Needs analysis}

It can be seen from the descriptive statistics that there are serious problems of school adaptation in the leftbehind children in grades 4 to 6 , mainly in the aspects of psychological and emotional adaptation, which hinder the development of relationships with classmates and teachers, and lead to poor interpersonal adaptation. Through six group group tutoring :" friend, hello "," who 
am I "," I am the best "," goodbye, bad mood "," achieve a better self "," my harvest "gradually lead to improve the interpersonal communication and psychological emotional adaptation of left-behind children.

\subsubsection{Effectiveness evaluation}

Group effect assessment form assesses group activities from interpersonal and psychological emotional aspects. Among them very do not accord with mark 1, compare do not accord with mark 2, general mark 3, compare accord with mark 4, very accord with mark 5. According to the data of the eight team members, the total score of the eight team members was higher than 4 points, indicating that their self-confidence had been greatly improved, their interpersonal skills had also improved, and all the team members had expressed great interest in the group activity. And from the data before and after the scale, the score of the post-test was significantly higher than that of the pre-test, and the adaptation of the group members was obviously improved, indicating that the effect of group activities was obvious.

Table 2. Comparison of Interpersonal Relationship and Psychological Emotional Dimensions

\begin{tabular}{|c|c|c|c|c|c|}
\hline & Group & $\begin{array}{c}\text { Number of } \\
\text { cases }\end{array}$ & $\begin{array}{c}\text { Average } \\
\text { value }\end{array}$ & $\begin{array}{l}\text { Standard } \\
\text { deviation }\end{array}$ & Sig \\
\hline \multirow[t]{2}{*}{$\begin{array}{c}\text { Tota } \\
1\end{array}$} & $\begin{array}{l}\text { Pre-activity } \\
\text { testing }\end{array}$ & 8 & 17.0000 & 2.39046 & 0.000 \\
\hline & $\begin{array}{l}\text { Post-activity } \\
\text { testing }\end{array}$ & 8 & 35.0000 & 1.19523 & \\
\hline
\end{tabular}

\section{REFLECTION AND CONCLUSION}

\subsection{Reflection}

There are six activities in the current period. Although the number of activities is relatively small compared with some other groups, it has been completed well and has basically achieved the expected goal, but due to the limitation of manpower, material resources and time, There are many shortcomings in the process of activities to be improved. Because of the near end of the period and the limitation of various conditions, the activity time is relatively short. When the group members enter the activity state, the social workers have to end in time and carry out the next activity, which also has an impact on the effect of the group activities. In addition, it is difficult for social workers to coordinate and grasp the details at the same time because they are both hosts, leaders, observers and reporters. Therefore, it is necessary to strengthen their own ability to carry out practice. Because they only pay attention to the accumulation of social work related theories and ignore the accumulation of social work practice during their study in school, it is particularly difficult to carry out practical work.

\subsection{Conclusion}

The adaptation of left-behind children's schools affects the socialized development of children, and the school is another important activity place for left-behind children, so it needs extensive attention. There are not only families, society, but also schools that affect the healthy growth of left-behind children. Using the theory of social work professional knowledge to interfere with the adaptation of left-behind children's schools is conducive to enhancing the adaptability of left-behind children, enhancing their self-confidence, and further improving the maladjustment of left-behind children. It is found that the self-confidence and interpersonal ability of the group members have been obviously improved before and after the group activities, which indicates the feasibility and effectiveness of the group activities intervention. Through six group activities, to a certain extent, they improve their bad psychology and bad emotions, promote them to form a healthy psychology, is conducive to better adapt to school life, better adapt to social life.

\section{REFERENCES}

[1] Zhang Fangyi, Gong Huoliang. A Review on School Adaptation of Domestic Left-behind Children [J].] in China Journal of Baoding University 31(04):8995.

[2]Ladd G W,Kochenderfer B J,Coleman C C.Classroom peer acceptance,friendship,and victimization : distinct relational systems that contribute uniquely to children's school adjustment[J]. Child development, 1997.

[3] Zou Hong. A Study on Peer Acceptance, Friendship and School Adaptation [J].].10 Psychological Development and Education ,1997.

[4] Chen Keyan. Group Work Intervention Study on the Improvement of Peer Relationship among Leftbehind Children [D].]; and Jiangxi University of Finance and Economics.

[5] Zhou Zongkui, Sun Xiaojun, Liu Ya, et al. J]. on Psychological Development and Education of Rural Left-behind Children Journal of Beijing Normal University: Social Sciences Edition ,2005.

[6] Yang Qin. Discussion on the Education of Leftbehind Children in Rural Areas [D].] Wuhan: central China normal University.

[7] Cao Shurong. Empirical Study on School Adaptation of Rural Left-behind Children —_ Taking 304 leftbehind children in 6 counties of Hubei Province as an example [J].].1 Youth Exploration ,2006.

[8] Xiang Yinhui. D]. on the Practice and Reflection of 
Social Work in the Growth of Left-behind Children in Rural Areas Northwestern University ,2013.

[9] Valentina Mazzucato, Victor Cebotari, Angela Veale, Allen White, Marzia Grassi, Jeanne Vivet.International parental migration and the psychological well-being of children in Ghana, Nigeria, and Angola[J]. Social Science \&Medicine, 2015.

[10] Li Jinghui. Problems and Countermeasures of School Adaptation for Left-behind Children in Rural Primary Schools — Taking Dongguang School in Wangqing County, Jilin Province as an example [J].].1 Modern Educational Science ,2014.

[11] Fan Xianzo. [J].] Problems and Countermeasures in Rural "Left-behind Children" Education Journal of the National Institute of Educational Administration ,2005.

[12] Zhou Yulin. Group Work Intervention Study on Improving the Interpersonal Ability of Rural Leftbehind Children [D].] in China Northwest normal University ,2018.

[13] Yang Yulan. A Study on School Adaptation of Rural Left-behind Children [D].] Central China normal University.

[14] Liu Wanlun. Wo Jianzhong. [J].] Relationship between Teacher-student Relationship and Adaptability of Primary and Middle School Students Psychological Development and Education ,2005(1):87-90. 